Dear author,

Please note that changes made in the online proofing system will be added to the article before publication but are not reflected in this PDF.

We also ask that this file not be used for submitting corrections. 


\title{
The concept of margins of stability can be used to better understand a change in obstacle crossing strategy with an increase in age
}

\author{
Laura Hak $^{\text {a }}$, Florentina J. Hettinga ${ }^{\text {b }}$, Kim R. Duffy ${ }^{\text {, }}$, Jo Jackson ${ }^{\text {b }}$, Gavin R.H. Sandercock ${ }^{\text {, }}$, \\ Matthew J.D. Taylor ${ }^{\mathrm{b}, *}$ \\ ${ }^{a}$ Research Institute MOVE, Department of Human Movement Sciences, Faculty of Behavioural and Movement Sciences, VU University Amsterdam, the Netherlands \\ ${ }^{\mathrm{b}}$ School of Sport, Rehabilitation and Exercise Sciences, University of Essex, Colchester, United Kingdom
}

\section{A R T I C L E I N F O}

\section{Article history:}

Accepted 20 December 2018

Available online $\mathrm{xxxx}$

\section{Keywords:}

Gait

Obstacle crossing

Margins of stability

Older adults

\begin{abstract}
A B S T R A C T
The purpose of the current study was to use the margins of stability (MoS) to investigate how older adults choose between minimizing the risk of a forward fall when crossing an obstacle and the ease of maintaining forward progression during the steps taken behind the obstacle. In the current study 143 communitydwelling older adults aged between 55 and 83 years old, were divided into three age groups based on tertials of age. All participants were asked to complete five trials of obstacle walking and five trials of normal walking. For the trials of normal walking, the main difference between groups was that MoS at initial contact was lower in the older age groups. For the trials of obstacle crossing the MoS at the instants of obstacle crossing with both the leading and trailing limb became smaller with an increase in age. This result might imply that older people choose to use a strategy during obstacle crossing that results in smaller chance of falling forward if an obstacle was struck. A negative consequence of this more conservative strategy was a smaller MoS at the instants of initial contact after crossing the obstacle, thus a larger chance of a backward fall. These findings provide more insight into the regulation of stability during obstacle crossing and specifically in the differences in strategy between younger and older people, and therefore these results might be used for further research to investigate whether obstacle crossing strategies are trainable in older adults, which could be used as advisory programs aimed at fall prevention and/ or engagement in an active lifestyle.
\end{abstract}

(c) 2018 Published by Elsevier Ltd.

\section{Introduction}

Reduced stability during walking is common in older adults which may predispose individuals to a fall (Hausdorff et al., 1998; Maki, 1997; Tinetti et al., 1986). When crossing an obstacle the challenge to stability is likely to be increased and as such older adults use a more conservative strategy during obstacle crossing compared to younger adults (Tinetti et al., 1986; Park et al., 2013; Galna et al., 2009). However, it is not clear if this is actually effective in terms of compensating for a decrease in stability or will even further increase the risk of a fall. A conservative strategy results in a slower walking speed when crossing an obstacle, which in turn increases the time the centre of pressure (CoP) displaces under the trailing foot (Park et al., 2013). Consequently, the distance between CoP and centre of mass (CoM) at the instant of

\footnotetext{
* Corresponding Author at: University of Essex, School of Sport, Rehabilitation and Exercise Sciences, Wivenhoe Park, Colchester CO4 3SQ, United Kingdom.

E-mail address: mtaylor@essex.ac.uk (M.J.D. Taylor).
}

obstacle crossing is smaller resulting in decreased forward angular momentum. This will be advantageous as it increases the ability to recover if the obstacle is struck by allowing adequate placement of the foot after the obstacle (Pijnappels et al., 2005). This difference in obstacle crossing behavior could be seen as a strategy to compensate for the reduced dynamic stability of older adults. However, a potential disadvantage of this strategy is the ability to maintain appropriate forward progression during the steps directly following the obstacle which would result in an increased risk of losing balance in the backward direction especially when negotiating a slippery floor or uneven ground (Bhatt et al., 2005; Hak et al., 2013a). Therefore, for a proper understanding of the consequences of the change in obstacle crossing strategy with aging it is necessary to not only study the obstacle crossing maneuver itself, but also the steps directly following obstacle crossing.

The margin of stability (MoS) is a measure of dynamic stability during walking (Hof et al., 2005). The strength of this measure is that not only the position, but also the velocity of the CoM with respect to the base of support (BoS) is taken into account. This 
measure has been used to study gait stability during steady state walking and perturbed walking in people with and without gait impairments (Curtze et al., 2011; Hak et al., 2013c, 2013b, 2012; Hof et al., 2007; McAndrew Young et al., 2012). The MoS are used to better understand the consideration between minimizing the risk of a fall as a consequence of a trip when crossing an obstacle and the possibility to maintain forward progression after crossing the obstacle (Fig. 1). Walking speed is an important variable influencing the size of the MoS because a reduced walking speed will also reduce the forward velocity of the CoM. (Hak et al., 2013a). During unperturbed walking the extrapolated centre of mass (XCoM) lies typically anterior with respect to the leading foot at initial contact (Fig. 1). Strictly seen, the XCoM is now located outside the border of the base of support, and that is why walking might be seen as unstable in the forward direction. However, maintaining forward progression is a requirement for walking forward, and that is why, for example, Hof (Hof, 2008) defines a stable walking pattern as the placement of the CoP at a constant distance behind the XCoM. In the current paper we have followed this definition of a stable gait pattern, and therefore we have chosen to define the situation in which the XCoM is located anteriorly with respect to the heel of the leading foot, as the backward border of the base of support (BoS), as positive (Fig. 1). Note, that this definition of 'stable' is opposite to the mechanical definition of stability in the case of an inverted pendulum.

The purpose of the current study was to use the MoS to investigate how older adults choose between minimizing the risk of a forward fall when crossing an obstacle and the ease to maintain forward progression during the steps taken after the obstacle, and whether this choice changed with age. To fulfill this purpose, MoS was calculated at both the instants of obstacle crossing with the leading and trailing limb and at the consecutive initial contacts after the obstacle. We hypothesized that the MoS during both obstacle crossing and the subsequent initial contacts after the obstacle would be relatively small, indicating a conservative strategy, but would also hamper the maintenance of forward progression after the obstacle. Furthermore, the reductions in MoS would be greater with an increase in age. Lastly, we hypothesized that MoS at the instance of obstacle crossing would be correlated with MoS measured during subsequent steps of obstacle crossing. There may be common factors influencing MoS, fall-risk and differences in obstacle crossing strategies and these are likely to be correlated within repeated measures, although we expected that this correlation might become weaker when the amount of steps after obstacle crossing increases. At last, since walking speed in older adults declines year on year past the age of 60 years (Himann et al., 1988; Song and Geyer, n.d) we wanted to see if walking speed contributed to MoS during obstacle clearance.

\section{Methods}

\subsection{Participants}

A sample of 158 community-dwelling older adults (101 females, 57 males; $65.7 \pm 6.8 \mathrm{yr} ; 168.6 \pm 9.2 \mathrm{~cm} ; 74.0 \pm 14.8 \mathrm{~kg}$ ) participated (Table 1). Ethical approval (BS2014) was granted by the local university ethics committee and all participants gave informed consent. The inclusion criteria allowed for a representative sample of a community-dwelling older adults and was as follows; all participants lived independently and were independent walkers (able to walk at least $10 \mathrm{~m}$ unaided), with no surgical procedures within the last six months and aged fifty-five years old or older.

\subsection{Equipment}

A seven camera Vicon T20 (Oxford, UK) infrared motion capture system sampling at $100 \mathrm{~Hz}$ was used to undertake the threedimensional motion analysis. Prior to each data capture session, the Vicon system was calibrated and a residual of $<2 \mathrm{~mm}$ for each camera was accepted. Sixteen passive reflective markers were attached, using wig-tape, to landmarks of the lower body in accordance to Davis' lower body model (Davis et al., 1991).

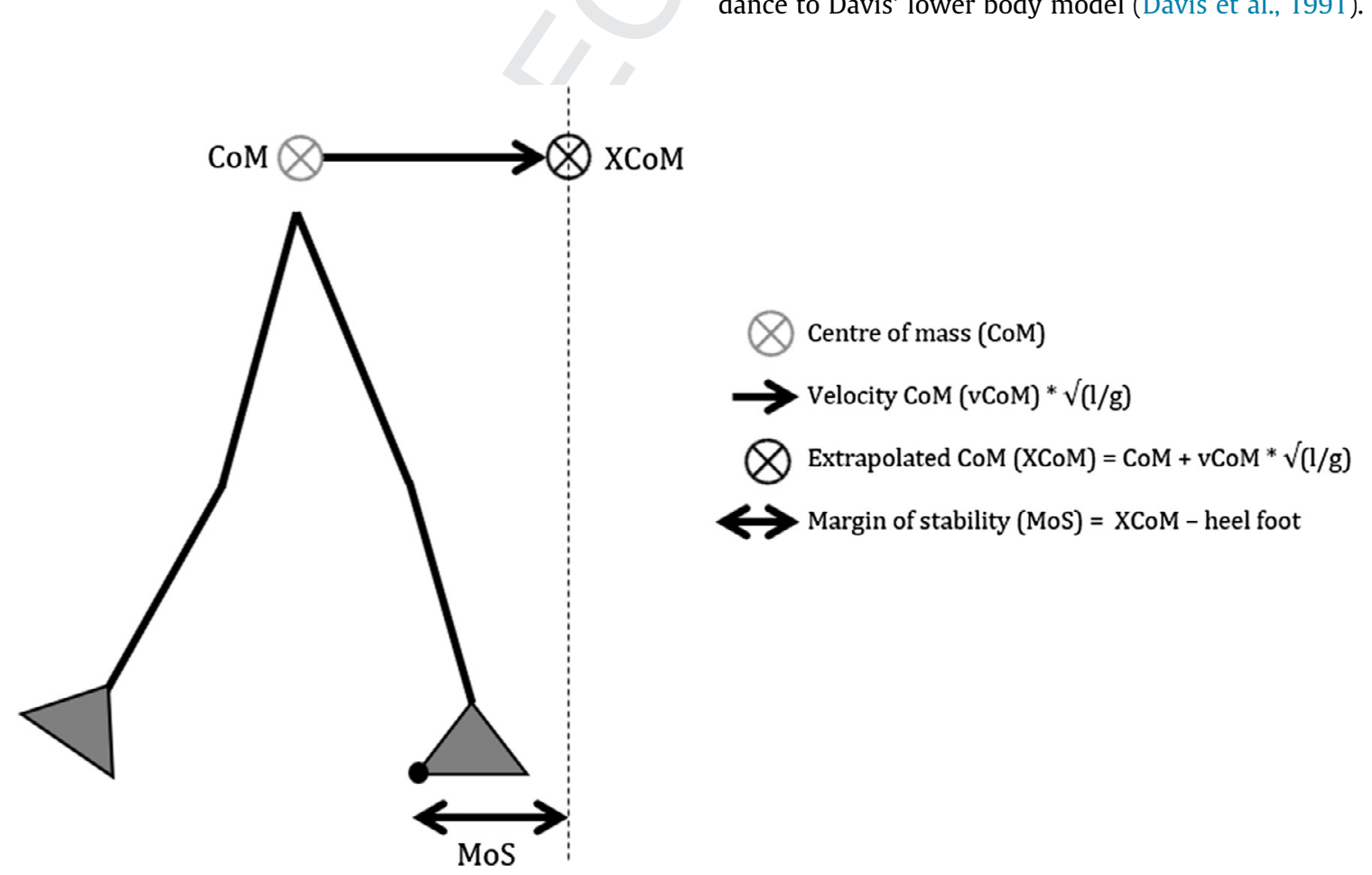

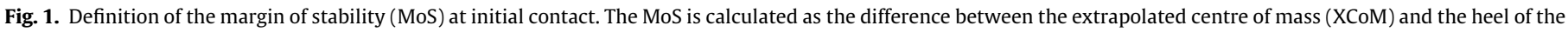
leading foot, in which the XCoM is defined as the position of the centre of mass (CoM) plus its velocity times $\sqrt{l / g}$. 
Table 1

Participant characteristics separated for the young, middle and old group.

\begin{tabular}{|c|c|c|c|}
\hline & $\begin{array}{l}\text { Younger } \\
\text { (55-62 years) }\end{array}$ & $\begin{array}{l}\text { Middle } \\
\text { (63-67 years) }\end{array}$ & $\begin{array}{l}\text { Older } \\
\text { (68-83 years) }\end{array}$ \\
\hline Participants (amount of males) & $44(14)$ & $48(17)$ & $51(19)$ \\
\hline Age (years; Mean (SD) & $58.6(2.6)$ & $65.0(1.3)$ & $72.3(3.8)$ \\
\hline Height (cm; Mean (SD) & $169.4(9.4)$ & $168.4(8.9)$ & $168.0(8.7)$ \\
\hline Mass (kg; Mean (SD) & $73.1(15.6)$ & $74.7(15.2)$ & $73.35(12.8)$ \\
\hline
\end{tabular}

\subsection{Data collection}

For both normal walking and the obstacle clearance task participants were asked to walk at their comfortable walking speed in their own footwear. Two pairs of Brower timing gates (Utah, USA) were positioned ( $2.28 \mathrm{~m}$ apart) in the middle of a $10 \mathrm{~m}$ walkway and were used to calculate the walking speed. For the obstacle (Reebok Stepper - $100 \times 16 \times 40 \mathrm{~cm}$ ) clearance task the obstacle was placed in between the timing gates, in the middle of the $10 \mathrm{~m}$ walkway. Reflective markers were placed on the top 4 corners of the obstacle. Participants walked towards the obstacle at their comfortable walking speed and stepped over the obstacle in a manner of their choosing. No instructions were given with regard to which leg was the leading or trailing limb. Five trials per walking task were captured for each participant.

\subsection{Data analysis}

Processing of all trials was performed using Vicon Nexus ( $\mathrm{v}$ 1.8.5, Oxford, UK). Reconstruction of the markers and autolabelling of marker trajectories were performed. Each trial was then visually inspected and unlabelled marker trajectories were manually labelled. Gaps in marker trajectories of up to 10 sample frames were joined with linear interpolation filtered with a quintic spline filter (Woltring; mean square error of 10). Then low-pass filtered at $10 \mathrm{~Hz}$ using a 4th order Butterworth filter. This cut-off frequency was selected to attenuate noise without distorting highfrequency marker movement at heel contact (Sinclair et al., 2013). The marker trajectories were exported as a .csv file.

To calculate the MoS, CoM position was estimated as the average position of the markers attached at the left and right superior and anterior iliac spines, in line with previous studies (i.e. Hak et al., 2012; McAndrew Young et al., 2012). MoS was calculated in the anterio-posterior direction and was calculated at the instants of a maximum of three initial contacts during the trials of normal walking, and for the trials of obstacle crossing at the instants of (1) the instant at which the leading foot crossed the obstacle, (2) initial contact of the leading foot behind the obstacle (step1), (3) the instant at which the trailing foot crossed the obstacle, (4) initial contact of the trailing foot behind the obstacle (step2), and (5) an additional initial contact of the leading foot (step3) (Fig. 2). The instants of initial contact were defined as the instants at which the difference between the heel marker and the average position of the markers attached at the pelvis reached its maximum. The instants of obstacle crossing were defined as the instants at which the difference between the marker attached at the heel of the leading foot or the trailing foot and the average position of the four markers attached at the obstacle was equal to zero.

MoS was derived from the procedure introduced by Hof (Hof et al., 2005). The MoS was calculated as the position of the XCoM minus the border of the base of support (BoS). The border of the BoS was defined as the position of the heel marker attached at the leading foot at the instances of initial contact and the instance at which the trailing foot was crossing the obstacle and the posi- tion of the heel marker attached at the trailing foot at the instance at which the leading foot was crossing the obstacle. The extrapolated centre of mass (XCoM) was calculated as the CoM position plus its velocity times a factor $\sqrt{ }(1 / g)$, with 1 being the maximal height of the estimated CoM and $g$ the acceleration of gravity. Although similar, our method differs from that of Hof (Hof et al., 2005) who used force plate data instead of kinematic data for calculating the XCoM and the MoS.

\subsection{Statistical analysis}

To compare outcomes by age we created three equally-sized groups; $55-62$ years $(n=45) 63-67$ years $(n=48)$ and $68-83$ years $(n=51)$.

One-way ANOVA (Scheffe post hoc test) were used to test whether MoS and walking speed differed between age groups. Effect sizes were calculated testing whether the outcome measures differed between age groups.

To test the other hypotheses of this study, Generalized Estimating Equations (GEE) were used. GEE is a regression analysis technique that accounts for the dependency of the repeated measurements. A stationary dependent 3-dependent correlation matrix was chosen to adjust for the dependency of the three steps taken behind the obstacle. In case p-values were below 0.05 the regression coefficient $(\beta)$ was considered as significant. This analysis was performed using IBM SPSS Statistics 20.0.

A Regression equation was determined to test our hypothesis that the MoS measured at initial contact of the steps behind the obstacle (MoS_IC) are related to the MoS measured at the instants of crossing the obstacle with the leading leg (MoS_lead) and trailing leg (MoS_trail). To test our hypothesis that this correlation might become weaker when the number of steps after obstacle crossing increases, the repeated measurements within the data were restructured to create two single variables, namely a variable that compares the MoS at the first IC with the MoS at the second IC after crossing the obstacle (MoS_Step2) and a variable that compares the MoS at the first IC with the MoS at the third IC after crossing the obstacle (MoS_Step3). We adjusted models for age and normal walking speed. We calculated the exponents (standard errors) and derived the fully-adjusted estimated marginal means for each measure.

\section{Results}

A total of 143 subjects fulfilled a maximum of 5 trials of normal walking and 5 trials of obstacle walking. Means and standard deviations and effect sizes for the MoS and walking speed are displayed in Table 2 for each age group separately. MoS differed significantly between groups for the instants at which the leading leg crossed the obstacle $(\mathrm{F}=4.904 ; \mathrm{p}=0.010)$, the trailing leg crossed the obstacle ( $\mathrm{F}=4.287 ; \mathrm{p}=0.016$ ) and the second and third initial contacts behind the obstacle $(F=7.472 ; \mathrm{p}<0.01$ and $\mathrm{F}=5.759$; $\mathrm{p}<0.01)$. Walking speed differed between groups for obstacle walking $(F=4.724 ; p=0.01)$. Outcome measures printed in bold/ 


\begin{tabular}{|c|c|c|c|c|c|}
\hline Normal walking & \multicolumn{5}{|c|}{ Obstacle crossing } \\
\hline Initial contact (IC) & Obstacle lead & IC step 1 & Obstacle trail & IC step 2 & IC step 3 \\
\hline$\bigwedge$ & & &
\end{tabular}

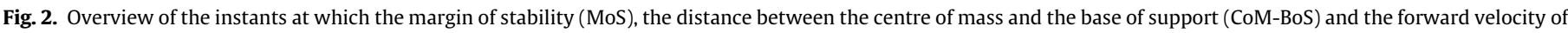

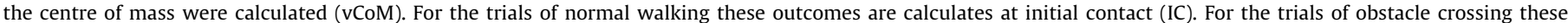

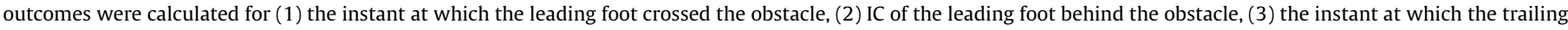
foot crossed the obstacle, (4) IC of the trailing foot behind the obstacle, and (5) an additional IC of the leading foot.

Table 2

Outcome measures (mean (SD)) and effect sizes (ES) calculated during initial contact (IC) of normal walking and different instants of the obstacle crossing.

\begin{tabular}{|c|c|c|c|c|c|c|c|}
\hline & All & Young (55-62 y) & Middle (63-67 y) & Older (68-83 y) & ES Young vs. Middle & ES Middle vs. Older & ES Young vs. Older \\
\hline \multicolumn{8}{|c|}{ Walk speed $\left(m \cdot s^{-1}\right)$} \\
\hline Normal & $1.34(0.18)$ & $1.38(0.18)$ & $1.36(0.19)$ & $1.30(0.16)$ & $\mathrm{d}=0.11$ & $d=0.34$ & $d=0.47$ \\
\hline Obstacle & $1.19(0.22)$ & $1.24(0.21)$ & $1.21(0.19)$ & $1.12(0.19)^{1}$ & $d=0.11$ & $d=0.47$ & $d=0.60$ \\
\hline \multicolumn{8}{|l|}{$\mathrm{MoS}$} \\
\hline IC Normal & $0.15(0.06)$ & $0.16(0.06)$ & $0.15(0.05)$ & $0.13(0.06)$ & $d=0.25$ & $d=0.36$ & $d=0.67$ \\
\hline Obstacle lead & $0.48(0.13)$ & $0.50(0.11)$ & $0.50(0.14)$ & $0.39(0.14)^{1,2}$ & $\mathrm{~d}=0.00$ & $d=0.79$ & $d=0.88$ \\
\hline IC Step 1 & $-0.03(0.16)$ & $-0.04(0.08)$ & $-0.03(0.07)$ & $-0.05(0.09)$ & $d=0.13$ & $d=0.25$ & $\mathrm{~d}=0.12$ \\
\hline Obstacle trail & $0.38(0.08)$ & $0.40(0.15)$ & $0.40(0.14)$ & $0.36(0.19)^{2}$ & $\mathrm{~d}=0.00$ & $d=0.35$ & $d=0.33$ \\
\hline IC Step 2 & $0.17(0.07)$ & $0.19(0.07)$ & $0.17(0.07)$ & $0.13(0.06)^{1,2}$ & $d=0.29$ & $d=0.62$ & $d=0.92$ \\
\hline IC Step 3 & $0.16(0.07)$ & $0.16(0.07)$ & $0.16(0.06)$ & $0.11(0.07)^{1,2}$ & $\mathrm{~d}=0.15$ & $d=0.92$ & $d=0.71$ \\
\hline
\end{tabular}

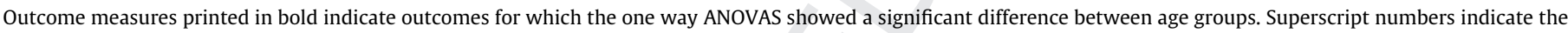

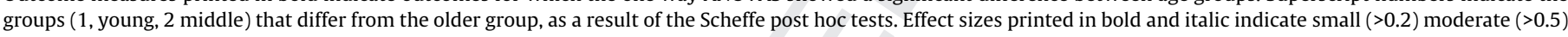
or large (>0.8) effect sizes between age groups.

Table 3

Results (regression coefficients $(\beta)$ and standard errors (SE)) of the Generalized Estimating Equation (GEE)

\begin{tabular}{lll}
\hline & MoS_IC: & \\
\cline { 2 - 3 } & $\beta$ & $(\mathrm{SE})$ \\
\hline Intercept & 0.217 & $(0.017)$ \\
Lead & 0.518 & $(0.155)$ \\
Trail & 0.416 & $p<.001$ \\
& & $(0.151)$ \\
Step 2 & 0.190 & $p<.001$ \\
Step 3 & 0.176 & $0.016)$ \\
Age & & $(0.017)$ \\
Walking speed & -0.002 & $p<.001$ \\
& & $(0.007)$ \\
& 0.194 & $p=.338$ \\
& & $(0.037)$ \\
& & $p<.001$ \\
\hline
\end{tabular}

The generalized equation used: $\quad$ MoS_IC $=\beta 1 \cdot \operatorname{MoS} \_L e a d+\beta 2 \cdot$ MoS_Trail $+\beta 3 \cdot$ MoS_Step $2+\beta 4 \cdot$ MoS_Step $3+\beta 5^{*}$ age $+\beta 5^{*}$ speed + intercept.

italic indicate moderate to large effect sizes for outcomes between age groups.

In Table 3 the outcome of the GEE is given. MoS measured at initial contact of the steps behind the obstacle appeared to be positively and significantly related to the MoS calculated at the instants at which the leading and trailing leg crossed the obstacle. The MoS for both the second and third initial contacts behind the obstacle were significantly larger compared to the MoS at the first initial contact behind the obstacle. The disturbance following the obstacle was greater for obstacle lead and obstacle trial phases compared to IC step 1 phase and that this tended to reduce the further away from the obstacle (steps 2 and 3). Walking speed contributed significantly to the size of the MoS and, finally, age appeared not to be a significant covariate.

\section{Discussion}

This study used the MoS to study balance regulation during obstacle crossing. More specifically the purpose of the current study was to investigate how older adults choose between minimizing the risk of a forward fall when crossing an obstacle and the ease to maintain forward progression during the steps taken behind the obstacle. For a proper understanding of the behavior when crossing an obstacle, MoS was also measured during normal walking. The MoS and walking speed during normal walking appeared to reduce with age; however this difference did not reach the level of significance (small to moderate effect size). This result suggests that the chance of an interruption of forward walking, and in extreme cases the necessity to make a recovery step to prevent a backward fall might increase, but is small during normal walking.

For the trials of obstacle crossing we found differences between age groups in MoS for both the instants of crossing the obstacle with the leading and trailing limb. The MoS at these instants became smaller with an increase in age, and was especially reduced in the oldest group, which was in line with our hypothesis. This result might imply that older people choose to use a strategy during obstacle crossing that results in a smaller chance of falling forward if contact is made with the obstacle. A negative consequence of this more conservative strategy might be smaller MoS at the instants of initial contact after crossing the obstacle, seen by the significant relationship we have found between MoS measured at the instants of obstacle crossing and the instants of initial 
contact behind the obstacle. Smaller MoS at the initial contacts suggests a larger risk of falling backward in case of for example a (unexpected) slippery floor or uneven surface.

For the first initial contact of the leading limb after crossing the obstacle we have found small and even negative MoS for all age groups, which did not appear to differ between age groups. These MoS around zero at initial contact indicate that all age groups use a strategy that results in a minimum MoS necessary to continue walking after crossing the obstacle. The MoS has to be at least positive at the instant of toe off following the IC of the first step after crossing the obstacle (IC step 1), otherwise forward progression will be hampered and a loss of balance in the backward direction will result (Hof, 2008; Pai and Patton, 1997). To realize this, the XCoM should be replaced actively in anterior direction during the double support phase. Based on the results of the GEE, it might be that the lower walking speed, resulting in a lower forward velocity of the CoM seems to be an important cause for this smaller MoS at the first initial contact after crossing the obstacle.

At IC step 2 and IC step 3 after the obstacle, MoS was much larger compared to the IC step 1, and were even comparable to the MoS measured during normal walking. For these steps MoS differed between age groups (large effect size); MoS was, as expected, smaller for the older subjects, especially for the oldest subjects, compared to the younger subjects. This smaller MoS for the older subjects was mainly due to a lower walking speed, causing a smaller forward velocity of the CoM. Differences in MoS for the second and third step between the 'older' and the 'younger' group were respectively 0.06 and $0.05 \mathrm{~m}$. These differences can be considered as clinically relevant, as these differences are comparable to differences found between amputees and non-amputees (Hak et al., 2013c) and are even larger than differences measured between able-bodied people and people after a stroke (Hak et al., 2013b).

An important limitation of this study is that we did not collect data of the steps before crossing the obstacle. Looking at a number of steps before the obstacle would be an added value because it will show how gait is modulated in anticipation of the obstacle, especially in cases of pre-planned obstacle clearance, like the task we used in the current study. For the crossing maneuver and the steps following obstacle crossing data were missing. However, the missing data were random between groups, based on the results of a Little's MCAR Test, and that a sufficient number of data were left, due to the large number of participants. Furthermore, it is important to realize that the participants in this study were all independent community dwelling individuals who had no history of falls. A prospective study might be useful to investigate whether the variables measured in the current study, especially the MoS, predict the chance of falls. Another useful addition might study the behavior of obstacle crossing in clinical populations who are prone to falls. Lastly, we calculated the position of the CoM as the average of the four markers attached to the pelvis instead of taking the whole body CoM. This might negatively influence the accuracy of the results. However, based on a post-hoc analysis for ten randomly selected participants, where we used a fullbody marker set, it appeared that the difference in MoS was small between these methods (within one standard deviation), in line with differences previously found (Whittle, 1997), and comparable between the different instants of obstacle crossing, and therefore conclusions based on the results would not be different for these two methods. An advantage of estimating the CoM based on only the markers attached to the pelvis is the reduced amount of markers necessary to calculate the MoS.

To conclude, subjects choose an obstacle avoidance strategy resulting in a negative $\operatorname{MoS}$ at first initial contact following the obstacle maneuver, implying that an active replacement of the $\mathrm{XCoM}$ was necessary during the double support phase to continue forward progression. For the second and third step after the obsta- cle, MoS were much larger, and even comparable with the MoS during normal walking. Older subjects seem to use a more conservative strategy when crossing the obstacle, which was in line with previous studies (Galna et al., 2009; Park et al., 2013; Pijnappels et al., 2005). This conservative strategy is reflected in a smaller MoS at the instants of crossing the obstacle with the leading and trailing limb and a smaller MoS for the second and first step after the obstacle. This results in a smaller chance of falling forward if an obstacle is struck, but also in a larger chance of a backward fall for the steps directly after crossing the obstacle. Although the differences found during the obstacle crossing trial between age groups could also be the direct result of the reduced physical capacities with an increase in age, the results found in the current study seem to confirm our hypothesis that elderly choose an obstacle crossing strategy that minimizes the risk of a trip as a result of an obstacle hit. Secondly, the results found in the current study confirm that, for a proper understanding of the risks during obstacle crossing in elderly, not only changes in the crossing maneuver itself but also for the first two steps directly following this crossing maneuver should be taken into account. These results might be of use for further research to investigate whether obstacle crossing strategies are trainable in elderly or are simply a result of a reduced physical ability. Results of such a study might be used within training and advisory programs aimed at fall prevention and/or engagement in an active lifestyle, preferably in a safe setting in which older people can practice obstacle crossing at different walking speeds, resulting in different MoS.

\section{Conflict of interest}

LH, FJH, KRD, JJ, GRHS, MJDT have no potential conflicts of interest.

\section{Uncited references}

Blake et al. (1988), Campbell et al. (1981), Chou and Draganich (1997), Downton and Andrews (1991), Martin et al. (2008), Prudham and Evans (1981), Stalenhoef et al. (2002), Tinetti and Speechley (1989), Tinetti et al. (1988).

\section{Acknowledgments}

There was no additional funding to perform the current study.

\section{References}

Bhatt, T., Wening, J.D., Pai, Y.-C., 2005. Influence of gait speed on stability: recovery from anterior slips and compensatory stepping. Gait Posture 21, 146-156. https://doi.org/10.1016/j.gaitpost.2004.01.008.

Blake, A.J., Morgan, K., Bendall, M.J., Dallosso, H., Ebrahim, S.B., Arie, T.H., Fentem, P. H., Bassey, E.J., 1988. Falls by elderly people at home: prevalence and associated factors. Age Ageing 17, 365-372.

Campbell, A.J., Reinken, J., Allan, B.C., Martinez, G.S., 1981. Falls in old age: a study of frequency and related clinical factors. Age Ageing 10, 264-270.

Chou, L.S., Draganich, L.F., 1997. Stepping over an obstacle increases the motions and moments of the joints of the trailing limb in young adults. J. Biomech. 30, 331-337.

Curtze, C., Hof, A.L., Postema, K., Otten, B., 2011. Over rough and smooth: amputee gait on an irregular surface. Gait Posture 33, 292-296. https://doi.org/10.1016/ j.gaitpost.2010.11.023.

Davis, R.B., Õunpuu, S., Tyburski, D., Gage, J.R., 1991. A gait analysis data collection and reduction technique. Hum. Mov. Sci. 10, 575-587. https://doi.org/10.1016/ 0167-9457(91)90046-Z.

Downton, J.H., Andrews, K., 1991. Prevalence, characteristics and factors associated with falls among the elderly living at home. Aging Milan Italy 3, 219-228.

Galna, B., Peters, A., Murphy, A.T., Morris, M.E., 2009. Obstacle crossing deficits in older adults: a systematic review. Gait Posture 30, 270-275. https://doi.org/ 10.1016/j.gaitpost.2009.05.022.

Hak, L., Houdijk, H., Beek, P.J., van Dieën, J.H., 2013a. Steps to take to enhance gait stability: the effect of stride frequency, stride length, and walking speed on local 
dynamic stability and margins of stability. PLoS ONE 8,. https://doi.org/10.1371/ journal.pone.0082842 e82842.

Hak, L., Houdijk, H., Steenbrink, F., Mert, A., van der Wurff, P., Beek, P.J., van Dieën, J. H., 2012. Speeding up or slowing down?: Gait adaptations to preserve gait stability in response to balance perturbations. Gait Posture 36, 260-264. https://doi.org/10.1016/j.gaitpost.2012.03.005.

Hak, L., Houdijk, H., van der Wurff, P., Prins, M.R., Mert, A., Beek, P.J., van Dieën, J.H., 2013b. Stepping strategies used by post-stroke individuals to maintain margins of stability during walking. Clin. Biomech. 28, 1041-1048. https://doi.org/ 10.1016/j.clinbiomech.2013.10.010.

Hak, L., van Dieën, J.H., van der Wurff, P., Prins, M.R., Mert, A., Beek, P.J., Houdijk, H., 2013c. Walking in an unstable environment: strategies used by transtibial amputees to prevent falling during gait. Arch. Phys. Med. Rehabil. 94, 21862193. https://doi.org/10.1016/j.apmr.2013.07.020.

Hausdorff, J.M., Cudkowicz, M.E., Firtion, R., Wei, J.Y., Goldberger, A.L., 1998. Gait variability and basal ganglia disorders: stride-to-stride variations of gait cycle timing in Parkinson's disease and Huntington's disease. Mov. Disord. 13, 428437. https://doi.org/10.1002/mds.870130310.

Himann, J.E., Cunningham, D.A., Rechnitzer, P.A., Paterson, D.H., 1988. Age-related changes in speed of walking. Med. Sci. Sports Exerc. 20, 161-166.

Hof, A.L., 2008. The 'extrapolated center of mass' concept suggests a simple control of balance in walking. Hum. Mov. Sci. 27, 112-125. https://doi.org/10.1016/j. humov.2007.08.003.

Hof, A.L., Gazendam, M.G.J., Sinke, W.E., 2005. The condition for dynamic stability. J. Biomech. 38, 1-8. https://doi.org/10.1016/j.jbiomech.2004.03.025.

Hof, A.L., van Bockel, R.M., Schoppen, T., Postema, K., 2007. Control of lateral balance in walking. Gait Posture 25, 250-258. https://doi.org/10.1016/ j.gaitpost.2006.04.013.

Maki, B.E., 1997. Gait changes in older adults: predictors of falls or indicators of fear? J. Am. Geriatr. Soc. 45, 313-320. https://doi.org/10.1111/j.15325415.1997.tb00946.x.

Martin, F., Husk, J., Foster, N., Ballinger, C., Spencer-Williams, M., 2008. Older People's Experiences of Falls and Bone Health Services.
McAndrew Young, P.M., Wilken, J.M., Dingwell, J.B., 2012. Dynamic margins of stability during human walking in destabilizing environments. J. Biomech. 45 1053-1059. https://doi.org/10.1016/j.jbiomech.2011.12.027.

Pai, Y.-C., Patton, J., 1997. Center of mass velocity-position predictions for balance control. J. Biomech. 30, 347-354. https://doi.org/10.1016/S0021-9290(96) 00165-0.

Park, S., Ko, Y.-M., Park, J.-W., 2013. The correlation between dynamic balance measures and stance sub-phase COP displacement time in older adults during obstacle crossing. J. Phys. Ther. Sci. 25, 1193-1196. https://doi.org/10.1589/ jpts.25.1193.

Pijnappels, M., Bobbert, M.F., van Dieën, J.H., 2005. Push-off reactions in recovery after tripping discriminate young subjects, older non-fallers and older fallers. Gait Posture 21, 388-394. https://doi.org/10.1016/j.gaitpost.2004.04.009.

Prudham, D., Evans, J.G., 1981. Factors associated with falls in the elderly: a community study. Age Ageing 10, 141-146.

Sinclair, J., Taylor, P.J., Hobbs, S.J., 2013. Digital filtering of three-dimensional lower extremity kinematics: an assessment. J. Hum. Kinet. 39, 25-36. https://doi.org/ 10.2478/hukin-2013-0065.

Song, S., Geyer, H., n.d. Predictive neuromechanical simulations indicate why walking performance declines with ageing. J. Physiol. https://doi.org/10.1113/ JP275166.

Stalenhoef, P., Diedericks, J., Knottnerus, J., Kester, A., Crebolder, H., 2002. A risk model for the prediction of recurrent falls in community-dwelling elderly: a prospective cohort study. J. Clin. Epidemiol. 55, 1088-1094.

Tinetti, M.E., Franklin Williams, T., Mayewski, R., 1986. Fall risk index for elderly patients based on number of chronic disabilities. Am. J. Med. 80, 429-434 https://doi.org/10.1016/0002-9343(86)90717-5.

Tinetti, M.E., Speechley, M., 1989. Prevention of falls among the elderly. N. Engl. J. Med. 320, 1055-1059. https://doi.org/10.1056/NEJM198904203201606.

Tinetti, M.E., Speechley, M., Ginter, S.F., 1988. Risk factors for falls among elderly persons living in the community. N. Engl. J. Med. 319, 1701-1707. https://doi. org/10.1056/NEJM198812293192604.

Whittle, M.W., 1997. Three-dimensional motion of the center of gravity of the body during walking. Hum. Mov. Sci. 16, 347-355. https://doi.org/10.1016/S01679457(96)00052-8. 